\title{
A HET search for planets around evolved stars
}

\author{
Andrzej Niedzielski ${ }^{1,2}$ and Alex Wolszczan ${ }^{2,1}$ \\ ${ }^{1}$ Toruń Centre for Astronomy, Nicolaus Copernicus University \\ Gagarina 11,87-100 Toruń, Poland \\ ${ }^{2}$ Dept. of Astronomy \& Astrophysics, The Pennsylvania State University, \\ 525 Davey Laboratory, University Park, 16802 PA, USA
}

\begin{abstract}
We present our ongoing survey of 1000 GK-giants with the 9.2-m Hobby-Eberly Telescope in search for planets around evolved stars. The stars selected for this survey are brighter than $11 \mathrm{mag}$ and are located in the section of the HR-diagram, which is approximately delimited by the main sequence, the instability strip, and the coronal dividing line. We use the High Resolution Spectrograph to obtain stellar spectra for radial velocity measurements with a $4-6 \mathrm{~m} \mathrm{~s}^{-1}$ precision. So far, the survey has discovered a planetary-mass companion to the K0-giant HD 17092, and it has produced a number of plausible planet candidates around other stars. Together with other similar efforts, our program provides information on planet formation around intermediate mass main sequence-progenitors and it will create the experimental basis with which to study dynamics of planetary systems around evolving stars.
\end{abstract}

Keywords. Extrasolar planets, red giants, radial velocity

\section{Introduction}

Precision radial velocity (RV) studies have established more than a decade ago that GK-giant stars exhibit RV variations ranging from days to many hundreds of days (e.g. Walker et al. 1989, Hatzes \& Cochran 1993, Hatzes \& Cochran 1994). Enough observational evidence has been accumulated to identify three distinct sources of this variability, namely stellar pulsations, surface activity and a presence of substellar companions. A possibility to discover planets around post-MS giants, in numbers comparable to the current statistics of planets around MS-dwarfs (e.g. Butler et al. 2006), offers a very attractive way to provide the much needed information on planet formation around intermediate mass MS-progenitors $\left(\geqslant 1.5 M_{\odot}\right)$ and to create a foundation for studies of the dynamics of planetary systems orbiting evolving stars (e.g. Duncan \& Lissauer 1998).

Fourteen planet discoveries around GK-giants have been reported so far (Niedzielski et al. 2007, and references therein, Lovis \& Mayor 2007, Johnson et al. 2007). Locations of giants with planets in the HR diagram are shown in Fig. 1. All detections have been made using the Doppler velocity technique with the RV precision ranging from $\sim 5$ to $\sim 25$ $\mathrm{m} \mathrm{s}^{-1}$, exploiting the availability of many narrow absorption features generated in the cool atmospheres of evolved stars. These developments demonstrate that sufficiently large surveys of post-MS giants should soon furnish enough planet detections to meaningfully address the above problems.

Initial analyses based on the currently available statistics (Lovis \& Mayor 2007, Johnson et al. 2007) suggest that the frequency of massive planets is correlated with stellar mass. Because more massive stars probably have more massive disks, these results appear to support the core accretion scenarios of planet formation (Pollack et al. 1996). Furthermore, Pasquini et al. 2007 have used the apparent lack of correlation between 
the frequency of planets around giants and stellar metallicity to argue that this effect may imply a pollution origin of the observed planet frequency - metallicity correlation for main sequence stars (Fischer \& Valenti 2005). Finally, for planets around giants, the absence of planets on tight orbits can be explained as the effect of post-MS evolution of their parent stars, but, as discussed by Johnson et al. (2007), other scenarios must also be considered. For example, the observed paucity of small orbital radii can be the result of faster depletion of disks around more massive stars, as suggested by simulations carried out by Burkert \& Ida (2007) .

In this paper, we describe our contribution to searches for planets around post-MS stars with a survey of 1000 GK-giants with the 9.2-m Hobby-Eberly Telescope. Our program has already discovered a number of interesting planet candidates, first of which has been recently published by Niedzielski et al. (2007).

\section{The survey}

Our long-term project to search for planets around evolved stars with the 9.2-m HobbyEberly Telescope (Ramsey et al. 1998) and its High Resolution Spectrograph (Tull 1998) has been established in early 2004. The sample of stars we have been monitoring is composed of two groups, approximately equal in numbers. The first one falls in the "clump giant" region of the HR-diagram (Jimenez et al. 1998), which contains stars of various masses over a range of evolutionary stages. The second group comprises stars,

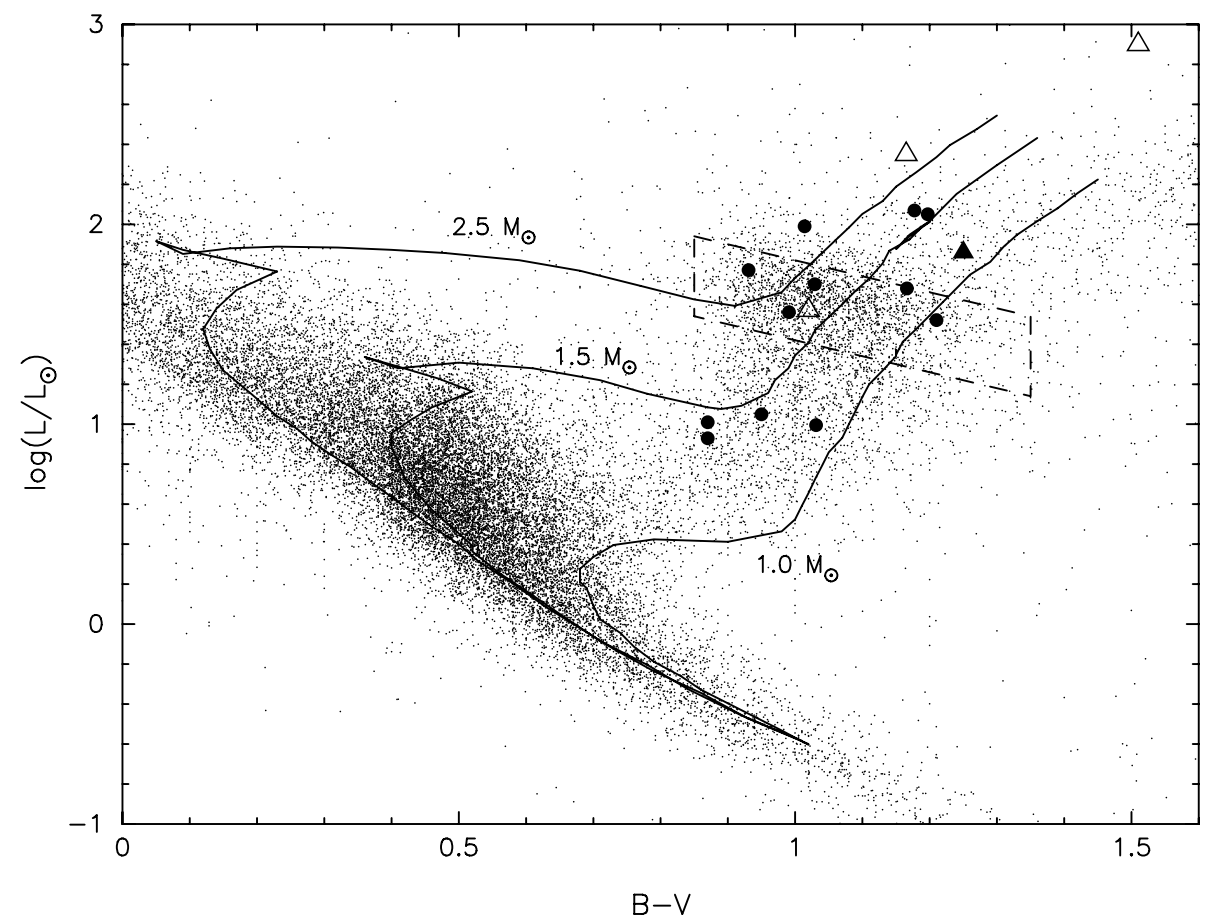

Figure 1. HR diagram for stars brighter than $V=10$ observable with the HET. The solid lines are evolutionary tracks from Girardi et al. (1996) for $\mathrm{Z}=0.02$. The dashed box delineates the red giant clump region (Jimenez et al. 1998). Symbols mark the stars with published planet detections and planets from this survey discussed in the text. (Filled triangle) Niedzielski et al. (2007); (Open triangles) unpublished detections from this survey; (Filled circles) other published detections. The diagram is based on data from the Hipparcos catalogue. 
which have recently left the MS and are located $\sim 1.5$ mag above it. Generally, as shown in Fig. 1, all our targets, a total of $\sim 1000$ GK-giants brighter than $\sim 11$ mag, occupy the area in the HR-diagram, which is approximately defined by the MS, the instability strip, and the coronal dividing line (a narrow strip in the HR-diagram marking the transition between stars with steady hot coronae and those with cool chromospheric winds Linsky \& Haisch 1979).

The HET observations and data analysis for this survey have been described by Niedzielski et al. (2007). Briefly, we observe with the HET in its queue-scheduling mode and use the HRS at the $\mathrm{R}=60,000$ resolution with the gas cell $\left(I_{2}\right)$ inserted in the optical path. In our target selection, we avoid bright objects, which are accessible to smaller telescopes. Consequently, more than $66 \%$ of our target stars are fainter than $\mathrm{V}=8$ mag. The observing scheme follows the standard practices implemented in precision radial velocity measurements with the iodine cell (Marcy \& Butler 1992). The spectral data used for RV measurements are extracted from the 17 echelle orders, which cover the 505 to $592 \mathrm{~nm}$ range of the $I_{2}$ cell spectrum. The observing strategy consists of the initial set of measurements of a target star (2-3 exposures, typically 3-6 months apart), to check for any RV variability exceeding a $30-50 \mathrm{~m} \mathrm{~s}^{-1}$ threshold, followed by more frequent observations, if a significant variability is detected. If the RV variability is confirmed, the star becomes part of the high priority list.

Radial velocities are measured by means of the commonly used $I_{2}$ cell calibration technique (Butler et al. 2006). A template spectrum is constructed from a high-resolution Fourier Transform Spectrometer (FTS) $I_{2}$ spectrum and a high signal-to-noise stellar spectrum measured without the $I_{2}$ cell. Doppler shifts are derived from the least-squares fits of template spectra to stellar spectra with the imprinted $I_{2}$ absorption lines. The average radial velocity for each epoch is calculated as a mean value of the independent determinations from the 17 usable echelle orders. The corresponding uncertainties of these measurements are estimated assuming that errors obey the Student's t-distribution. Typically, they fall in the $4-5 \mathrm{~m} \mathrm{~s}^{-1}$ range at $1 \sigma$-level. Radial velocities are referred to the Solar System barycenter using the Stumpff (1980) algorithm, which is accurate enough given the RV precision limitations that are intrinsic to the evolved stars.

As the intrinsic variability may contribute to the observed RV variations (e.g. Gray 2005), stellar line profiles are studied in detail in search for any signatures of a rotation induced spot activity. Also the existing photometry databases like Hipparcos, Tycho or Northern Variability Sky Survey (Woźniak et al. 2004) are used to study possible integrated light variations that might be interpreted as a result of pulsations. These analyses are reviewed elsewhere (Niedzielski et al. this vol.).

\section{Results}

In almost four years of observations, we have obtained more than one RV measurement for $>600$ GK-giant stars with a $4-6 \mathrm{~m} \mathrm{~s}^{-1}$ precision. Adopting a working definition of RV scatter $\leqslant 40 \mathrm{~m} \mathrm{~s}^{-1}$ for a stable (single) red giant, we find that $55 \%$ of stars in that sample are single, $20 \%$ are new binaries and $25 \%$ stars possibly have low-mass companions.

We have been currently monitoring more than 30 planetary candidate companion stars and have obtained preliminary orbital solutions for most of them. Each one obviously requires a thorough examination of stellar activity, which includes bisector analysis and a study of $\mathrm{H}_{\alpha}$ variations. The RV curves and the corresponding best-fit orbital models for our first published planet around the K0 giant, HD 17092 (Niedzielski et al. 2007), and for three other examples of the detections that are being prepared for publication, are shown in Fig. 2. The observed RV curves are highly repeatable and their periods are not 
reproduced in the measured line bisector and photometric variations. Provisional stellar mass estimates using Girardi et al.(2000) evolutionary tracks indicate a planetary nature of the companions. It is quite clear that star 162 has a third, long-period companion, whose nature will be established in the course of further observations. The star 37 planet has the most compact orbit among the existing detections $(\mathrm{a}=0.6 \mathrm{AU})$, whereas the planet around star 18 may be orbiting the most massive star in the existing sample (5.5 $\left.M_{\odot}\right)$ and has an exceptionally high intrinsic RV noise.

A steadily increased number of stars observed in this survey makes it possible to carry out statistical studies of RV noise properties of GK-giants. Our preliminary results confirm the intrinsic RV jitter of red giants with the maximum of its distribution at about $20 \mathrm{~m} \mathrm{~s}^{-1}$. Furthermore, the RV scatter increases with B-V, easily reaching $100 \mathrm{~m} \mathrm{~s}^{-1}$ for stars later than K5. Clearly, more observations are needed to understand the nature of the scatter, part of which may be contributed by short-period pulsations, which remain unresolved by the sparse sampling of our survey.

Searches for planets around evolved stars are still in their infancy compared to similar programs for solar-type stars, which have been steadily furnishing new planet detections to bring the count up to over 250 at the time of this writing. However, it is the former searches that are now needed to obtain new information on stellar mass and timedependent aspects of planet formation and evolution that is not accessible through the latter ones. A continuation of the survey described in this proposal, together with other similar programs, is already creating a base of planet detections around GK-giants, which
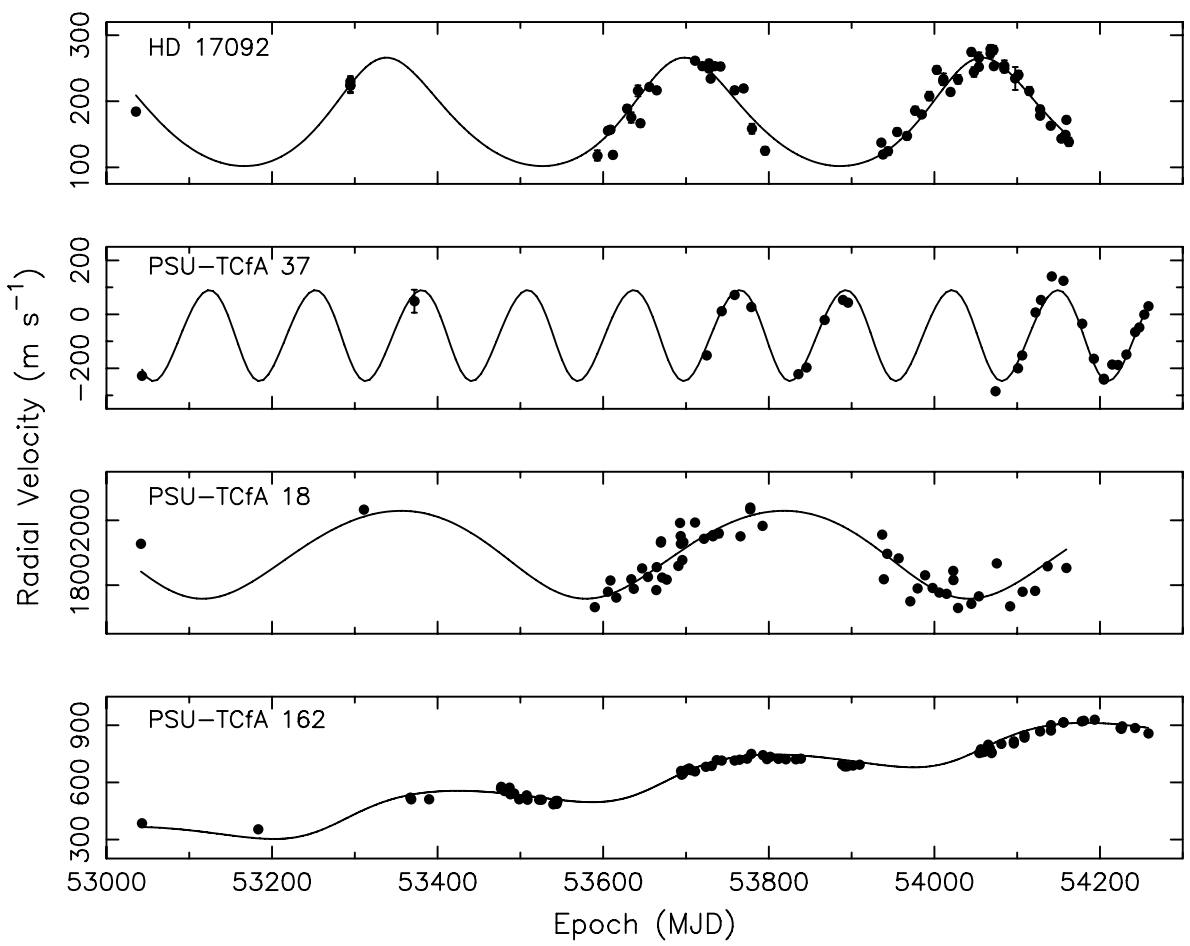

Figure 2. Radial velocity measurements (filled circles) and the best-fit orbital models (solid lines) for a sample of four K-giant stars monitored by the HET survey. For most data points, the circle size is larger than the sizes of error bars. 
will soon become sufficient to fully address the questions of stellar mass and chemical composition dependence of planet formation for masses $>1 M_{\odot}$ and of the possible fates of planetary systems under the influence of an evolving parent star. This knowledge will improve our understanding of the astrophysics of planetary systems, it will provide an experimental base for theories of the far future of the Solar System and it will broaden our knowledge of the astrophysical aspects of long-term survival of life on Earth and elsewhere, including a possibility of the emergence of life on planets in the expanded habitable zones of red giants (Lopez et al. 2005).

\section{Acknowledgements}

AN and AW were supported in part by the Polish Ministry of Science and Higher Education grant 1P03D 007 30. AW also acknowledges a partial support from the NASA Astrobiology Program. The Hobby-Eberly Telescope (HET) is a joint project of the University of Texas at Austin, the Pennsylvania State University, Stanford University, LudwigMaximilians-Universität München, and Georg-August-Universität Göttingen. The HET is named in honor of its principal benefactors, William P. Hobby and Robert E. Eberly.

\section{References}

Burkert, A., \& Ida, S. 2007, Astrophysical Journal, 660, 845

Butler, R. P., et al. 2006, Astrophysical Journal, 646, 505

Butler, R. P., Marcy, G. W., Williams, E., McCarthy, C., \& Dosanjh, P. 1996, Publications of the Astronomical Society of the Pacific, 108, 500

Duncan, M. J., \& Lissauer, J. J. 1998, Icarus, 134, 303

Fischer, D. A., \& Valenti, J. 2005, Astrophysical Journal, 622, 1102

Girardi, L., Bressan, A., Chiosi, C., Bertelli, G., \& Nasi, E. 1996, Astronomy \& Astrphysics Supplement Series, 117, 113

Girardi, L., Bressan, A., Bertelli, G., \& Chiosi, C. 2000, Astronomy \& Astrphysics Supplement Series, 141,371

Gray, D. F. 2005, Publications of the Astronomical Society of the Pacific, 117, 711

Hatzes, A. P., Cochran, W. D. 1993, Astrophysical Journal, 413, 339

Hatzes, A. P., \& Cochran, W. D. 1994, Astrophysical Journal, 422, 366

Jimenez, R., Flynn, C. \& Kotoneva, E. 1998, Monthly Notices of the Royal Astronomical Society, 299,515

Johnson, J. A., et al. 2007, Astrophysical Journal, 665, 785

Linsky, J. L., \& Haisch, B. M. 1979, Astrophysical Journal Letters, 229, L27

Lopez, B., Schneider, J., \& Danchi, W. C. 2005, Astrophysical Journal, 627, 974

Lovis, C., \& Mayor, M. 2007, Astronomy \&3 Astrphysics, 472, 657

Marcy, G. W. \& Butler, R. P. 1992, Publications of the Astronomical Society of the Pacific, 104, 270

Niedzielski, A., et al. 2007, Astrophysical Journal, 669, 1354

Pasquini, L., Döllinger, M. P., Weiss, A., Girardi, L., Chavero, C., Hatzes, A. P., da Silva, L., \& Setiawan, J. 2007, Astronomy \& Astrphysics, 473, 979

Pollack, J. B., Hubickyj, O., Bodenheimer, P., Lissauer, J. J., Podolak, M., \& Greenzweig, Y. 1996, Icarus, 124, 62

Ramsey, L. W., et al. 1998, Proceedings of the SPIE, 3352, 34

Stumpff, P. 1980, Astronomy \& Astrphysics Supplement Series, 41, 1

Tull, R. G. 1998, Proceedings of the SPIE, 3355, 387

Walker, G. A. H., Yang, S., Campbell, Bruce, I., Alan W. 1989 Astrophysical Journal, 343, 21

Woźniak, P. R., et al. 2004, Astronomical Journal, 127, 2436 